\title{
Body balance reduces eczema in stress-related atopic dermatitis
}

\author{
Anna Utterström, Solbritt Lonne-Rahm
}

Department of Medicine, Karolinska University Hospital, Solna, Stockholm, Sweden; sol-britt.lonne-rahm@,karolinska.se

Received 20 September 2009; revised 13 October 2009; accepted 14 October 2009.

\begin{abstract}
Atopic dermatitis (AD) is a common chronic, pruritic, relapsing inflammatory skin disease that affects about two to three percent of the adult population in Sweden. It is characterized by dry, itchy skin and is aggravated by stress. This study examined if relaxation through movement and body awareness can reduce stress and eczema in patients with $A D$. Nine patients with atopic dermatitis whose condition worsened due to stress were treated with a body balance relaxation method. Estimation of extent of skin lesions, pruritus, salivary cortisol, as an objective marker for chronic stress, and subjective stress, and DLQI, were performed. Of these parameters, the extent of skin lesions, pruritus, and the subjective stress were decreased as well as chronic stress. This study indicated that relaxation using body balance reduced stress and eczema in patients with stress-related atopic dermatitis.
\end{abstract}

Keywords: Atopic Dermatitis; Body Balance; Eczema; Relaxation; Stress.

\section{INTRODUCTION}

Atopic dermatitis (AD) is a common, pruritic, chronically relapsing inflammatory skin disease that is often associated with other atopic manifestations such as bronchial asthma, allergic rhino-conjunctivitis and food allergies. The etiology of AD is unknown, but it is presumed to be multifactorial with interaction between genetic and environmental factors [1]. The skin disease is typically present in early childhood and may continue or recur later. It affects about two to three percent of the adult population [2]. There is geographical variation in the prevalence of eczema in adults both within and between countries and it has become more prevalent in recent decades [3]. The diagnosis of atopic dermatitis is usually based on several variables, including anamnestic and clinical findings. The criteria of Hanifin and Rajka [4] have been scientifically evaluated and can be applied successfully in the clinical situation. Important trigger factors are irritants, microbial agents, food, hormones and stressful life events [5].

Emotional stress has a great influence on the immune system and can be manifested in skin disease [6]. A stress reaction is a nonspecific response of the organism to any type of burden or threat. A stimulus is perceived as stressful if the individual does not believe that he or she can exercise any influence on the frequency and intensity of the stimulus. It is a highly regulated, widespread reaction and a number of hormones are activated during a stress response.

Relaxation has been used to treat atopic eczema patients and dermatological treatment combined with massage $[7,8]$ and behavioral therapy has been reported to be an effective method [9].

The aim of the study was to examine a method of relaxation for this group of patients, who usually have problems finding a way to relax by themselves, in order to reduce chronic stress since stress is a worsening factor for patients with AD. Subjective stress was investigated by using a stress enquiry, and objective stress by using cortisol as an indicator of chronic stress [10].

\section{MATERIAL AND METHODS}

\subsection{Patients}

Nine women with $\mathrm{AD}$, with a mean age of 32 years (range: 19 to 42) who had active atopic moderate to severe dermatitis (as defined by the criteria of Hanifin and Rajka [4]) were referred to the Neurocutaneous reception at Karolinska University Hospital in Solna, Sweden, by other dermatologists. Each had a history of eczema being aggravated by stress.

The patients evaluated their ongoing stress level and the intensity of itching by using a Visual Analog Scale from 0 to $100(0=$ nothing, $100=$ worst $)$ before and after each treatment. They also evaluated the percentage of body surface covered by eczema by using a Visual Ana$\log$ Scale from 0 to 100 and completed a "Dermatology 
Life Quality Index" (DQLI) before and after the treatment period. Each patient received five individual treatments, once a week. Each session lasted approximately 1.5 hours. The patients have their usual topical treatment during the period of stress relaxation.

\subsection{Salivary Cortisol Test}

Salivary cortisol samples were obtained from all patients before and after the period of treatment. The saliva samples were collected in plastic vials at 8.00 am on three consecutive days. At $10.00 \mathrm{pm}$ on the last day, $0.25 \mathrm{mg}$ of dexametason was administered orally, followed by a new cortisol test on the following morning. The samples were stored at $-20^{\circ} \mathrm{C}$ until analysis and cortisol concentrations were determined using a radioimmunoassay kit (Spectria Cortisol, Orion Diagnostica, Espoo, Finland). The ratio of the mean of the previous three values to the last cortisol value was determined, with a low ratio being an indicator of chronic stress [7].

\subsection{The Dermatology Life Quality Index (DLQI)}

The DLQI consists of ten items covering different aspects of skin disease-related symptoms during the previous week. There are four alternative responses to each question: "not at all, "a little," "a lot" and "very much," with corresponding scores of $0,1,2,3$, respectively. The total score is calculated by adding the scores for each question, and the total score ranges from a minimum of 0 to a maximum of 30 , with a higher score indicating a greater impact on quality of life. The Danish version of the DLQI has previously been shown to have satisfactory reliability and validity [11]

\subsection{The Body Balance Relaxation Method}

The Body Balance relaxation method is a program of body awareness exercises designed to enhance mental and physical awareness. The patient becomes more aware of his or her body and emotions at the time of treatment, which enhances his or her sense of self awareness and relaxation. The exercises are done with the patient in sitting and recumbent positions, and the time required for the treatment is between sixty and ninety minutes.

The Body Balance relaxation method divides the body into seven different levels:

1) Pelvic floor, including legs and feet, 2) Pelvis, 3) Solar plexus, 4) Thoracic cage, 5) Neck, 6) Forehead and head, 7) Top of the head.

During the treatment, the patient is instructed to concentrate on one level at a time, focusing first on the pelvic floor in a sitting position and then the rest of the program while lying down, continuing level by level to the top of the head. Each level is treated as follows:

1) Movement: The patient makes slow cross-lateral movements and rotations along and around the center line of the body as well as movements between the upper and lower parts of the body.

2) Breathing: The patient is instructed to inhale and exhale in pace with the movements, and then to relax and breathe freely. The exercises stimulate abdominal breathing and the patient experiences a deepening of breathing and an increased feeling of roominess in his or her thoracic cage.

3) Sound: The patient also makes vowel sounds together with the movements, with different vowels used for each level, for example, an "o" sound for the pelvic floor.

4) Color: The patient is asked to visualize colors during the program, with one color used for each level, for example, visualizing red when concentrating on the pelvic floor.

Movements, breathing, sound and color help the patient focus on one level of the program at a time. Once the patient has focused on a particular part of the body, he or she is instructed to lie back and relax. During relaxation, the patient notes how the particular part of the body feels and how mentally and physically relaxed he or she is at that time. With practice, the patient can use this technique alone to achieve relaxation and inner balance as well as to improve self awareness.

\subsection{Statistical Methods}

The total sum of stress, skin symptoms and DQLI scores for patients in the group was compared using the Wilcoxon Signed Rank Test. The level of significance was set at $p<0.05$.

\section{RESULTS}

\subsection{Extent of Eczema}

The mean percentage of body surface covered by eczema before the first session was 46, range 10-90, and after the last session was mean 29 , range $10-60$, with a strong tendency to a decrease $(p=0.068)$ (Table 1 and 2).

\subsection{Itching}

Before the first session, the mean of itching scored on the Visual Analog Scale was 39, range 9-73, and after the last session 5 , range $0-23$, with a decrease $(p<0.01)$.

\subsection{Stress Score}

Before the first session, the mean of the subjective stress score was 46, range 18-79, and after the last session, mean 3, range $0-21$, with a decrease $(p<0.01)$.

\subsection{Cortisol Ratio}

The mean of the cortisol ratio before the first session 
Table 1. Before the treatment.

\begin{tabular}{cccccc}
\hline Patient & Extent & Pruritus & Stress & Cortisol ratio & DLQI \\
\hline & & 46 & 22 & 0.8 & 10 \\
& & 70 & 49 & 1.3 & 11 \\
4 & 22 & 24 & 0.8 & 19 \\
5 & 20 & 9 & 18 & 2.7 & 10 \\
6 & 1 & 80 & 32 & 0.4 & 2 \\
7 & 2 & 70 & 69 & 1.9 & 13 \\
8 & 3 & 60 & 63 & 0.2 & 4 \\
9 & 10 & 49 & 79 & 0.4 & 8 \\
Mean & $\mathbf{4 6}$ & $\mathbf{3 9}$ & $\mathbf{4 6}$ & $\mathbf{1 . 0}$ & 7 \\
\hline
\end{tabular}

Table 2. After the treatment.

\begin{tabular}{cccccc}
\hline Patient & Extent & Pruritus & Stress & Cortisol ratio & DLQI \\
\hline 1 & 30 & 7 & 0 & 2.0 & 5.2 \\
2 & 40 & 0 & 0 & 10.4 & 12 \\
3 & 60 & 23 & 3 & 6.9 & 17 \\
4 & 20 & 2 & 0 & 3.1 & 1 \\
5 & 20 & 0 & 21 & 2.0 & 17 \\
6 & 30 & 2 & 0 & 3.6 & 1 \\
7 & 20 & 0 & 0 & 5.7 & 10 \\
8 & 30 & 10 & 1 & 5.3 & 4 \\
9 & 10 & 0 & $\mathbf{3}$ & $\mathbf{4 . 9}$ & $\mathbf{9}$ \\
\hline
\end{tabular}

was 1 , range $0-3$, and after the last session, mean 5 , range $2-10$. Thus, there was an increase $(p<0.01)$, indicating a lower level of chronic stress following treatment.

\subsection{DLQI}

Before the first session, the mean of DLQI was 9, range 4-19, and after the last session being 9, range 1-17, with no evident difference.

\section{DISCUSSION}

Personality and stress may play important roles in the pathogenesis of AD [12]. AD can be induced or worsened by stress, and all of the patients involved in the study confirmed that stress is an initiating or exacerbating factor. We do not know exactly how many patients, indicating that stress impairs their eczema, but all patients with AD who seeking to Karolinska Hospital may offer to go on neurocutana reception, where stress management is part of the treatment. The atopic dermatitis outpatients at the Neurocutaneous reception were referred in order to reduce their chronic stress levels. Some of the patients had tried different types of relaxation before without success. All together, the components of the program helped patients to reduce stress and increase physical and mental awareness. In this limited study in which women with stress-related atopic eczema participated, we have shown that this type of relaxation has tended to reduce the inflammation of the skin. Unfortunately, during this period, no men participate in the study, but to reduce stress is important in the treatment of inflammatory diseases for all patient categories.

The body balance method stimulates several senses and gradually improves physical and mental awareness. The design of the movements and total focus on one part of the body at a time make the method meditative, so it is easy to let go of thoughts and stay focused on the body. The movements reduce muscular tension along the spine and provide physical relaxation. The patient also becomes more aware of the location of tension and tender points in the back and how they change during treatment. This increases the patient's awareness of what is happening in his or her body.

During treatment, it was observed that the patients' movements increased when they reported feeling stressed or worried while their movements slowed and became more rhythmical as they gradually began to relax. Relaxation was enhanced through the vocalization of sounds, which tended to reduce the tempo of the patients' movements, and coordination was enhanced through the synchronization of movement, breathing and sound.

All of the patients in the study reported a reduction in stress at each session and cited that they felt that they were centered in the core of their bodies. Seven of the nine patients reported that this feeling of being centered lasted for some time after the sessions, from a couple of 
hours up to several days.

One patient, who had been on sick leave for approximately one year due to burnout and who experienced bouts of anxiety especially in the evenings, found that she felt less stressed and anxious at each session and experienced less stress in daily life. She also reported that her skin did not itch or burn as much or as often as before. This patient was also able to reduce her anxiety outside of the sessions by using the body balance method. This positive effect was confirmed by an independent psychologist who had been seeing the patient for several months prior to the study.

The main symptoms of $\mathrm{AD}$ are dryness of the skin and severe pruritus. This occurs episodically or persistently in the eczematous areas and leads to vigorous scratching. Unconscious scratching may be particularly severe during the night, resulting in poor sleep. During the study, the patients became relaxed during the sessions and their skin reactions decreased. They reported less or no itching, burning or pain compared to before the sessions. They also reported that they were able to maintain this sense of relaxation for some time afterwards.

It is possible that the lack of impact on the DLQI might be due to the rather short time period of the study, with a latency in the recording of changes in this parameter. The patients may have adapted to their illness and had a negative self-esteem and they may have needed psychological counseling to reduce their avoidance and helplessness [10]. For this reason, it would be interesting to conduct a longer study on the body balance method.

In this uncontrolled study, we have focused on relaxation with a body balance method. Though there was no indication of an increased use of topical treatment, using steroids, within the studied patients, it would be useful to perform a similar controlled study.

\section{CONCLUSIONS}

Stress management is an important part of the treatment of stress-related eczema. Relaxation through body bal- ance may be a method to reduce subjective and objective stress and eczema in atopic eczema patients.

\section{REFERENCES}

[1] Schmied, C. and Saurat, J.H. (1991) Epidemiology of atopic eczema. In: Ruzicka T, Ring J, Przybilla B, eds. Handbook of Atopic Eczema., 9-14.

[2] Leung, D.Y.M. and Bieber, T. (2003) Atopic dermatitis. The Lancet, 361, 151-60.

[3] Croner, S., (1991) Atopic dermatitis-epidemiology. Pediatr Allergy Immunol, 2 (Suppl): 6-7.

[4] Hanifin, J.M. and Rajka, G. (1980) Diagnostic features of atopic dermatitis. Acta Derm Venereol (Stockh), 92(suppl.), 44-7.

[5] Morren, M.A., Przybilla, B., Bamelis, M., Heykants, B., Reynaers, A. and Degreef, H. (1994) Atopic dermatitis: triggering factors. J Am Acad Dermatol, 31, 467-73.

[6] Teshima, H., Kubo, C., Kihara, H., Imada, Y., Nagata, S., Ago, Y. and Ikemi, Y. (1982) Psychosomatic aspects of skin diseases from the standpoint of immunology. Psychother Psychosom., 37, 165-75.

[7] Schachner, L., Field, T., Hernandez-Reif, M., Duarte, A. and Krasnegor, J. (1998) Atopic dermatitis symptoms decreased in children following massage therapy. Pediat Dermatol, 15, 390-95

[8] Anderson, C., Lis-Balchin, M. and Kirk-Smith., M (2000) Evaluation of massage with essential oils on childhood atopic eczema. Phytother Res, 14, 452-56.

[9] Lange, S., Zschocke, I. and Amon, U. (1999) Augustin. Effects of combined dermatological and behavioral medicine therapy in hospitalized patients with psoriasis and atopic dermatitis. Hautarzt, 50, 791-97.

[10] Ljung, T., Andersson, B., Bengtsson, B.A., Björntorp, P. and Mårin, P. (1996) Inhibition of cortisol secretion by dexamethasone in relation to body fat distribution: a dose-response study. Obes Res, 4, 277-82.

[11] Zachariae, R., Zachariae, C., Ibsen, H., Mortensen, J. T. and Wulf, H. C. (2000) Dermatology life quality index: data from Danish inpatients and outpatients. Acta Derm Venereol (Stockh), 80, 272-76.

[12] Buske-Kirschbaum, Geiben, A. and Hellhammer, D. (2001) Psychobiological aspects of atopic dermatitis: an overview. Psychother Psychosom, 70, 6-16. 